\title{
Application and Practice of Problem-oriented Teaching Mode
}

\author{
Guizhou LV, Guanhui LIANG \\ Ordnance Engineering College, Department of Electronics and Optics Engineering, Hebei, Shijiazhuang \\ 050003
}

\begin{abstract}
In high-tech, students having extensive foundation knowledge, the course possessing rich knowledge point, and teaching content closely combining with obtain employment need, problem-oriented teaching mode is an important way to rationally organize the teaching content and improves teaching effect. In this paper, the problem-oriented teaching mode centers on the key links which need to grasp, combining with course instance, analyzing the following aspect: problem setting, role play of student and teacher and the strategy of enhancing the effect, summarizing the implementation of every link, and constructs an evaluation system of key link.
\end{abstract}

KEYWORD: problem-oriented; education system; evaluation system; strategy

In today, science and technology rapid developing, it is the requirement to create a "learning society, innovative society" for every society member. As a high-tech college for training innovative talents, it is an unavoidable issue about how to complete task of talent training under this demand so as to continuous provide talent according with society need. Problemoriented teaching mode dedicates to combine the condition needs of society with the experience in high-tech activity, and it is the aim to compare the learning motivation with learning effect, and to guide teaching operates efficiently[1].

\section{SOLVING THE PROBLEM IS THE CENTRAL TASK OF TEACHING ACTIVITY}

Teacher mainly teaches reason and technique and solving doubt. "Reason" and "technique" is the method to dispose question and the skill of solving problem, the starting point is the standpoint, culture, method and means of teacher. Doubt producing from student, it is the doubt and trouble and problem need to solve, which closely combines with keenly awareness of student. It is the important link to solve doubt for advancing learning effect and sense of achievement and enhancing learning confidence and ability.

Problem-oriented teaching method having a clear

Ordance Engineering College 2014 teaching research item: No. 2014JXJXLX034 direction, so the teacher and student can quickly solves problem, and understands knowledge, and advances ability in the process, obtaining better teaching effect only need paying less effort[2]. In the following, the using mode of the problem-oriented teaching mode is analyzed from the aspect of key link, organizing strategy and practice effect.

\section{THE KEY LINKS OF PROBLEM-ORIENTED TEACHING MODE}

\subsection{Knowledge point is start point of setting problem}

Any teaching method, its ultimate aim is to teach students a lot of knowledge point. Student successfully understanding knowledge point is the basic task of teaching, and effectively abstracting the knowledge point is the important link to complete teaching. In the process of abstracting knowledge point, the following content needs to be care: the first is embodying the course function in the process of training talent, the second is embodying the core content of the course, the third is embodying the student ability need and job position need.

Taking knowledge point abstracting of radar performance test for example, starting off knowledge point demand of radar entire machine, system and subsystem, abstracts the main knowledge point of this course and constructs the system frame diagram of knowledge, which making the teachers teaching content and student learning content clearly[3]. 


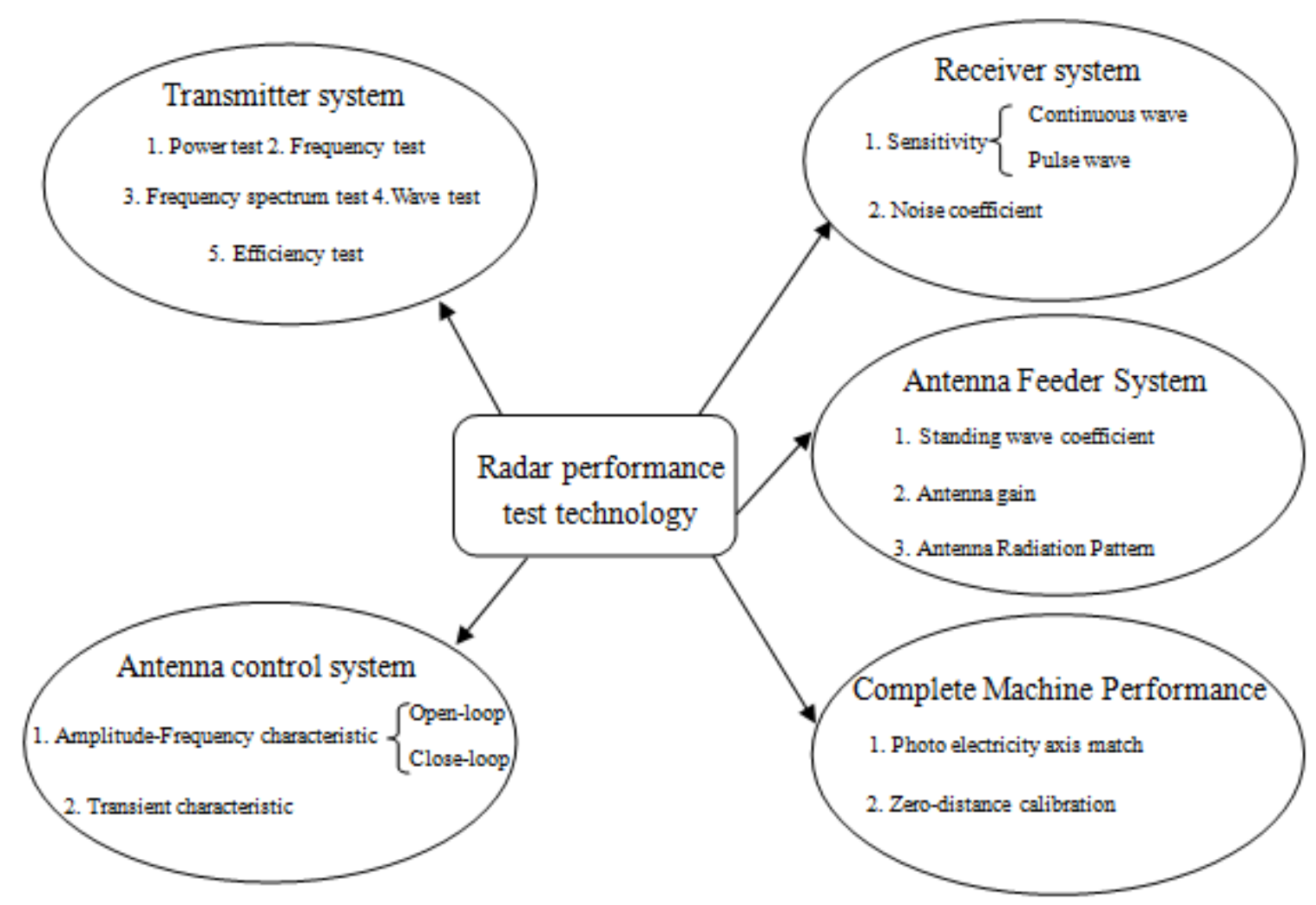

Figure.1 Knowledge point system structure of radar performance test technology

\subsection{Teacher is the leader of setting problem}

In teaching activities, "teaching" is input of "learning", the main responsibility body is teacher. The sentence of "If the teacher having great charm, the student will research deeply" in "The Analects" described the function and station of teacher thoroughly. It is the duty and obligation for teachers to set knowledge point to material problem which having specific purpose, highly operational and highly practical in problem-oriented teaching mode, so that the student can grasp knowledge point and obtain practical skill in the process of solving problem[4].

In the material practice of "radar performance test technology", it is important to actively explore the melting problem of knowledge point. First, melting the importance to problem; Second, melting the practice to problem; Third, melting the summarization to problem, consequently, student ability training flow which aims at every knowledge point is come into being as shown in Figure.2.

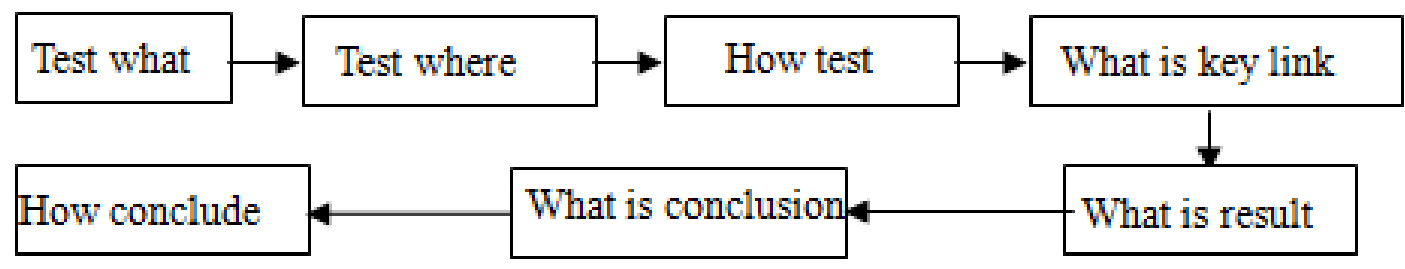

Figure. 2 Knowledge point training and exercise flow based on problem-oriented

\subsection{Student is administrator of solving problem}

Student is main body of teaching activity, the object of "teaching", the host of "learning", the center of teaching activity, the embody carrier of talent training. "Body" is afraid of immobility, brain being not afraid of using, hand is more and more flexible along with using, and brain is more and more clever along with using. In the practice process of problem- oriented teaching mode, only student active participate, initiator consider, practice in body can obtain better teaching effect and train excellent talent according with the need of job position.

In the course practice of "radar performance testing technology", surrounding the participate degree of thinking and activity, student executing ability appraisal system of synthetical practice subject is come into being as Table.1. 
Table.1 Appraisal standard chart of student solving problem ability

\begin{tabular}{|c|l|}
\hline No & \multicolumn{1}{|c|}{ Ability standard } \\
\hline 1 & Enthusiasm evaluation in process of solving problem \\
\hline 2 & Is the knowledge foundation of student aiming the problem abundant \\
\hline 3 & Is the idea of solving problem clear \\
\hline 4 & Practice ability of solving problem \\
\hline 5 & Harmonize, command, cooperation ability \\
\hline 6 & Summarize and "using one against three" ability \\
\hline
\end{tabular}

\subsection{Interaction of teacher and student advances the learning effect of solving problem}

Teaching activity is collision between thinking and thinking, communication between activity and activity, student and teacher should realizes knowledge transferring, ability training and diathesis enhancement. In "The Analects", much rhesis is collision spark as Confucius and his student discussing question and daily communication, incessant sedimentation in communication, finally coming into being immemorial wisdom, and becomes the philosophy of guiding our interaction with others.

In aspect of forming a good interactive atmosphere of teaching and learning, in the course teaching of "radar performance test technology", we pay attention to the followings: starting off the common knowledge foundation, setting the future job position need to guide, forming a good interactive atmosphere surrounding knowledge point. In practical implement, topology structure is adopted as Figure. 3.

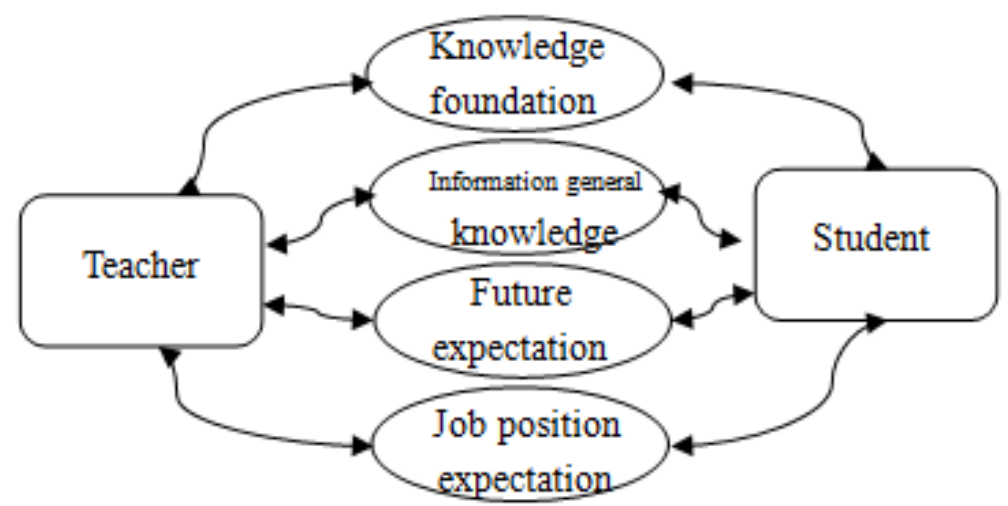

Figure.3 Implement strategy topological structure of better classroom tnteractivity

\section{CONCLUSION}

Problem-oriented teaching mode is of important guiding significance to teacher reasonable setting teaching link, completing teaching of knowledge, improving the enthusiasm and enhancing of learning and the pertinence of learning. Combining the teaching experience, analyzes the key link setting, disposing method and effect evaluation of problemoriented, which is of great guide importance to both teaching and learning, and which has some reference and promotional value in every level teaching process.

\section{REFERENCES}

[1] Min Zeng, Jinsong Xu. Implement appraise of problemoriented course design. Wireless Interconnected Science and Technology, 2011(3).

[2] Xiaoyong $\mathrm{Hu}$, Xiaoli $\mathrm{Hu}$, Dandan Xie. Research on problem-oriented strategies to promote online learners' cognitive skills. Journal of Distance Education, 2011(3).

[3] Yanheng Ma, Zhiyun Wang, Wenhua Hu, Zhiqiang Li. Radar performance test technology. National industrial Press, 2007.

[4] Chunli Zhou. Suggestion on Teaching Innovation of Colleges and Universities. Higher Education Research, 2010(12). 